\title{
Legal aspects of low-emission shipping in the light of provisions of "sulphur directive" adopted by the European Union
}

\author{
Beata Madejska, M. Sc., \\ Independent lawyer
}

\begin{abstract}

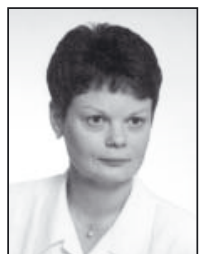

Shipping emits a variety of air pollutants: sulphur dioxide $\left(S O_{x}\right)$, nitrogen oxides $\left(N O_{x}\right)$, carbon dioxide $\left(\mathrm{CO}_{2}\right)$ and particulate matter PM. Air pollutant emissions from maritime transport can be transported over long distances and thus increasingly contribute to air quality problems. Key environmental regulations (international and European) coming into force in this decade address emissions of $\mathrm{SO}_{x}, \mathrm{NO}_{x}, \mathrm{CO}_{2}$ and $\mathrm{PM}$ to control and limit their impact in the atmosphere. In the European Union, accordingly to the legal regulations, in the sulphur emission control areas the required $S_{x}$ content of fuel will be reduced from $1.5 \%$ to $0.1 \%$ beginning January 2015. Globally, from 2020 onwards, ships operating in all other European Sea areas will have to use fuels with sulphur content of $0.5 \%$ or less.
\end{abstract}

Key words: ship emissions; sulphur dioxide; nitrogen oxides; sulphur directive; MARPOL Annex VI; protection of the environment; control on sulphur emissions

\section{BACKGROUND}

The main sources of air pollution are transport, power generation, industry, agriculture, and heating. All these sectors emit a variety of air pollutants - sulphur dioxide, nitrogen oxides, carbon dioxide and particulate matter. Shipping is a large and growing source of different kinds of atmospheric emissions. Air pollutant emissions from maritime transport can be transported over long distances and thus increasingly contribute to air quality problems in the European Union. Sulphur emissions are harmful to our health and to the wider environment, and need to be reduced. It is right that the shipping industry is being required to reduce its emissions in the way that other industries and transport sectors have already done. Current forecasts indicate that shipping in the EU will produce more emissions than all land-based sources by 2020 (source: Clean Air from Europe impact assessment, p. 31, 2005). Ship emissions contribute to acid rain (which damages crops and buildings), air pollution, ground-level ozone (smog), and marine eutrophication in the European Union. One of the European areas which is highly susceptible to the environmental impacts of ship emissions is the Baltic Sea.

The Baltic Sea with a total area of about $370,000 \mathrm{~km}^{2}$ is one of the world's largest brackish water basins, but it is also a small sea on a global scale. The Baltic is almost entirely land-locked and the water exchange is very limited. It is also a high priority for the EU, owning to its status as a Sulphur Emission Control Area, due to high pollution of the region. The Commission describes the Baltic Sea and also the North Sea and English Channel as "very fragile ecosystems", because of their significance for recognized ecological (unique ecosystem) and socio-economic reasons (recreation, tourism).

In order to achieve the desired effect on the environment, emissions regulations must be agreed and applied internationally.

\section{INTERNATIONAL LEGISLATION (IMO CONVENTION)}

The issue of controlling air pollution from ships, in particular, noxious gases from ships' exhausts was discussed in the lead up to the adoption of the 1973 MARPOL Convention. However it was decided not to include regulations concerning air pollution at the time.

Meanwhile, air pollution was being discussed in other arenas. The 1972 United Nations Conference on the Human Environment in Stockholm marked the start of active international cooperation in combating acidification, or acid rain. Between 1972 and 1977, several studies confirmed the hypothesis that air pollutants could travel several thousand kilometers before deposition and damage occurred. This damage includes effects on crops and forests. In 1979, a ministerial meeting on the protection of the environment, in Geneva, resulted in the signing of the Convention on Longrange Transboundary Air Pollution by 34 governments and the European Community. This was the first international legally binding instrument to deal with problems of air pollution on a broad regional basis (source: IMO website).

The MARPOL Convention is the main international convention covering prevention of pollution of the marine 
environment by ships from operational or accidental causes. It is a combination of two treaties adopted in 1973 and 1978, respectively, and also includes the Protocol of 1997 (Annex VI). It has been updated by amendments through the years.

The International Convention for the Prevention from Ships (MARPOL) was adopted on 2nd November 1973 at IMO and covered pollution by oil, chemicals, harmful substances in packaged form, sewage and garbage. The original Convention was signed on 17 February 1973, but did not come into force due to lack of ratifications. As a combination of 1973 Convention and the 1978 Protocol, the current MARPOL convention (commonly known as MARPOL 73/78) entered into force on 2 October 1983. As of May 2013, 152 states, representing 99,2 percent of the world's shipping tonnage, are parties to the convention.

Annex VI Prevention of Air Pollution from Ships entered into force $19^{\text {th }}$ May 2005. The revision of Annex VI was adopted in October 2008 and entered into force on $1^{\text {st }}$ July 2010.

The regulations in this Annex set limits on sulphur dioxide and nitrogen oxide, emissions from ship exhausts as well as particulate matter.

Annex VI contains provisions allowing for special Sulphur Emission Control Areas (SECAs) to be established with more stringent controls on sulphur emissions. In these areas the sulphur content of fuel oil used onboard ships must not exceed $1.5 \%$ by mass. Alternatively, ships must fit an exhaust gas cleaning system or use any other technological method to limit sulphur dioxide emissions.

The Baltic Sea Area is designated as a Sulphur Emission Control Area in the Protocol. The North Sea was adopted as Sulphur Emission Control Area in July 2005.

\section{EUROPEAN LEGAL PROVISIONS CONCERNING REDUCTION OF SHIP EMISSIONS}

At the European Union level there have been subsequent steps to regulate sulphur content in liquid fuels to reduce its emissions in the atmosphere during the past twenty years. Historically, the sulphur content of certain liquid fuels was regulated by Directive 93/12/EC, adopted in 1993 (Council Directive 93/12/EEC of 23 March 1993 relating to the sulphur content of certain liquid fuels, OJ 121, 11.5.1999), which placed restrictions on the marketing of diesel fuels used in road vehicles and gas oil used for off-road transport (but excluding aviation).

In the following years it has been deemed important to lay down limits for the sulphur content of other liquid fuels, in particular heavy fuel oil, marine gas oils and gas oils, on the basis of cost effectiveness studies and also in view of the regulation in Annex VI on sulphur content of marine fuel in the IMO's MARPOL Protocol of 1997. The result was the Sulphur Content of Liquid Fuels Directive No 32 adopted on 26 April 1999 (Council Directive 1999/32/EC of 26 April 1999 relating to a reduction in the sulphur content of certain liquid fuels and amending Directive 93/12/EEC). It established limits for sulphur content in heavy fuel oil $(1.0 \%$ after 1 st January $2003)$ and gas oil, including marine gas oil $\left(0.2 \%\right.$ after $1^{\text {st }}$ January 2000 and $0.1 \%$ after 1 st January 2008). In the latter case derogations for Greece throughout its territory, for Spain with regard to the Canary Islands, for France with regard to the French Overseas Departments, and for Portugal with regard to the archipelagos of Madeira and Azores were also included since the new limits may present technical and economic problems for these regions. In the Directive was also included a recommendation to continue the initiative to have the North Sea including English Channel declared as sulphur emission control area (ECA). The requirements concerning the sulphur content of gas oil and heavy fuel oil, heating oil and marine fuels appeared in the Directive to follow the incorporation into EU law of rules adopted by the International Maritime Organization. The incorporation of IMO standards aimed, inter alia, at reinforcing the restricted international monitoring and enforcement regime.

Member States were obliged to transpose the Directive into national legislation before $1^{\text {st }}$ June 2000.

The list of national enforcement measures in exemplary EU Member States, embracing the provisions of the Directive 93/12/EEC, are shown in the table below:

\begin{tabular}{|c|c|c|}
\hline Item & Country & National execution measures \\
\hline 1. & DENMARK & $\begin{array}{l}\text { 1. Bekendtgorelse af } 22 \text { juni } 2000 \text { om begraensning av svovindholdet I visse flydende braendstoffer } \\
\text { (Legal act: Bekendtgřrelse; Official Journal: Administrative measures) }\end{array}$ \\
\hline 2. & GERMANY & $\begin{array}{l}\text { 1. Dritte Verordnung zur Durchfuhrung des Bundes-Immissionsschutzgesetzes (Verordnung über den } \\
\text { Schwefelgehalt bestimmter flüssiger Kraft- oder Brennstoffe - } 3 \text { BlmSchV) vom 24/06/2002 BGbl. } \\
\text { Teil I n }{ }^{\circ} 41 \text { du 28/06/2002 p. } 2243 \text { (Legal act: Verordnung; Official Journal: Verwaltungsmassnahmen, } \\
\text { Entry into force: } 24 / 06 / 2002 \text {; Reference: (SG(2002)A/07440) } \\
\text { 2. Zweite Verordnung zur Änderung der Verordnung über die Beschaffenheit und die Auszeichnung } \\
\text { der Qualitäten von Kraftstoffen réf.: Bundesgesetzblatt I S. } 2243 \text { of } 28 \text { June 2002. Date d'adoption } \\
\text { de l'acte:24/06/02 (Legal act: Verordnung; Official Journal: Verwaltungsmassnahmen }\end{array}$ \\
\hline 3. & ESTONIA & $\begin{array}{l}\text { 1. Välisőhu kaitse seadus (Legal act: seadus, number: RTI, 19.05.2004, 43, } 298 \text {; Official Journal: } \\
\text { Elektrooniline Riigi Teataja, number: RTI, 19.05.2004, 43, 298, Entry into force: 30/09/2004; } \\
\text { Reference: (MNE(2004)52690) } \\
\text { 2. Säästva arengu seadus (Legal act: Legal act: seadus, number: RTI 1995, 31, 384; Official Journal: } \\
\text { Elektrooniline Riigi Teataja, number: RT I 1995, 31, 384, Entry into force: 01/01/2001; Reference: } \\
\text { (MNE(2003)54275) } \\
\text { 3. VÄLISÖHU KAITSE SEADUS (Legal act: seadus; Official Journal: Elektrooniline Riigi Teataja, } \\
\text { Publication date: 08/01/2004; Reference: (MNE(2003)55186) } \\
\text { 4. Vedelkütuse nöuetekohasuse töendamise protseduuride ja korra kinnitamine (Legal act: } \\
\text { Majandusministri määrus; Official Journal: Elektrooniline Riigi Teataja, Publication date: 01/11/1998; } \\
\text { Reference: (MNE(2003)54970) } \\
\text { 5. Tarbijakaitseseadus (Legal act: seadus, number: RTI, 15.03.2004, 13, 86; Official Journal: } \\
\text { Elektrooniline Riigi Teataja, number: RTI, } 15.03 .2004,13,86 \text {, Entry into force: } 15 / 04 / 2004 \text {; Reference: } \\
\text { (MNE(2003)55290) }\end{array}$ \\
\hline
\end{tabular}




\begin{tabular}{|c|c|c|}
\hline Item & Country & National execution measures \\
\hline 3. & ESTONIA & 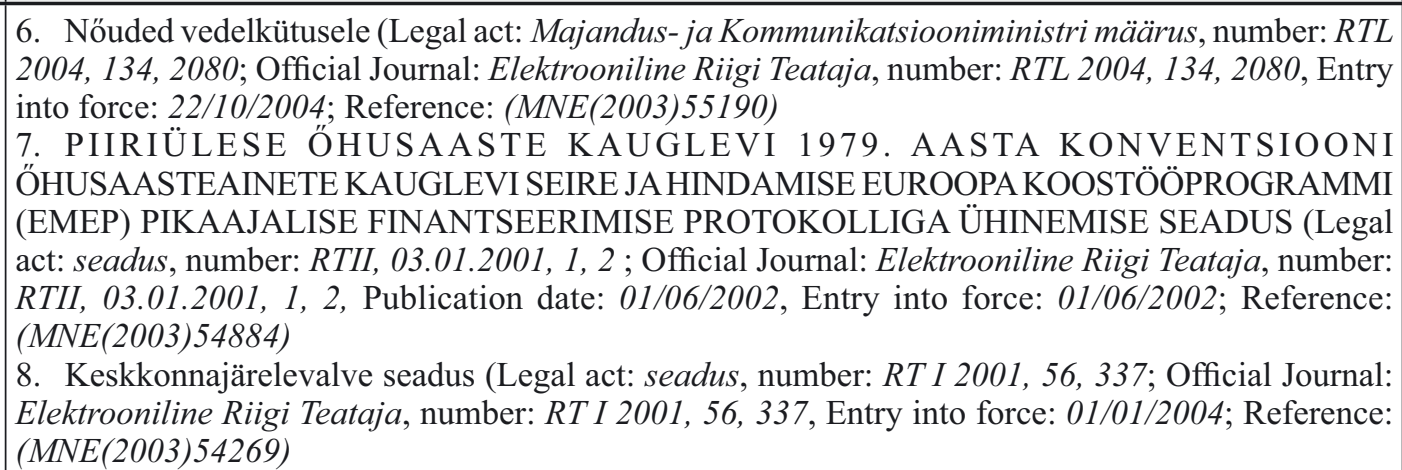 \\
\hline 4. & IRELAND & $\begin{array}{l}\text { 1. The Air Pollution Act (Sulphur Content of Heavy Fuel and Gas Oil) Regulations, } 2001 \text { S.I. } n^{\circ} 13 \\
\text { of 2001, Reference: } S G(2001) A / 01569)\end{array}$ \\
\hline 5. & FINLAND & $\begin{array}{l}\text { 1. Landskapslag om tillämpning i landskapet Aland av vissa riksförfattningar rörande atgärder mot } \\
\text { förorening av luften 02/04/1991 AFS Nr } 32 \text { de } 1991 \text { du 25/04/19991; SG(00)A/16230 du 03/01/2001 } \\
\text { et idem SG(2001)A/1657 du 07/02/2001 (Official Journal: Llands Författningssamling (ĹFS), number: } \\
\text { 32/1999, Publication date: } 25 / 04 / 1999 \text {, Entry into force: 02/04/1991) } \\
\text { 2. Alands Landskapsstyrelses Beslut om ändring av Alands landskapsstyrelses beslut om tillämpning } \\
\text { i landskapet Aland av vissa statsradsbeslut rörande atgärder mot förorening av luften 12/10/2000 } \\
\text { AFS Nr } 72 \text { de } 2000 \text { du 21/11/2000; SG(00)A/16230 du 03/01/2001 et idem SG(2001)A/1657 du } \\
07 / 02 / 2001 \text { (Official Journal: Ĺlands Författningssamling (ĹFS), number: } 72 / 2000 \text {, Publication date: } \\
\text { 21/11/2000, Entry into force: } 12 / 10 / 2000) \\
\text { 3. Valtioneuvoston asetus râskaan polttoöljyn ja kevyen polttoöljyn rikkipitoisuudesta }(766 / 200) \\
\text { 24/08/2000 Suomen Säädöskokoelma 30/08/2000 No 766, page 1986; SG(2001)A/1657 du 07/02/2001 } \\
\text { (Legal act: Valtioneuvoston asetus, number: } 766 / 200 \text {, Entry into force: } 24 / 08 / 2000) \\
\text { 4. Statsradets förodning nr } 766 \text { (Reference: }(S G(2000) A / 11718)\end{array}$ \\
\hline 6. & FRANCE & 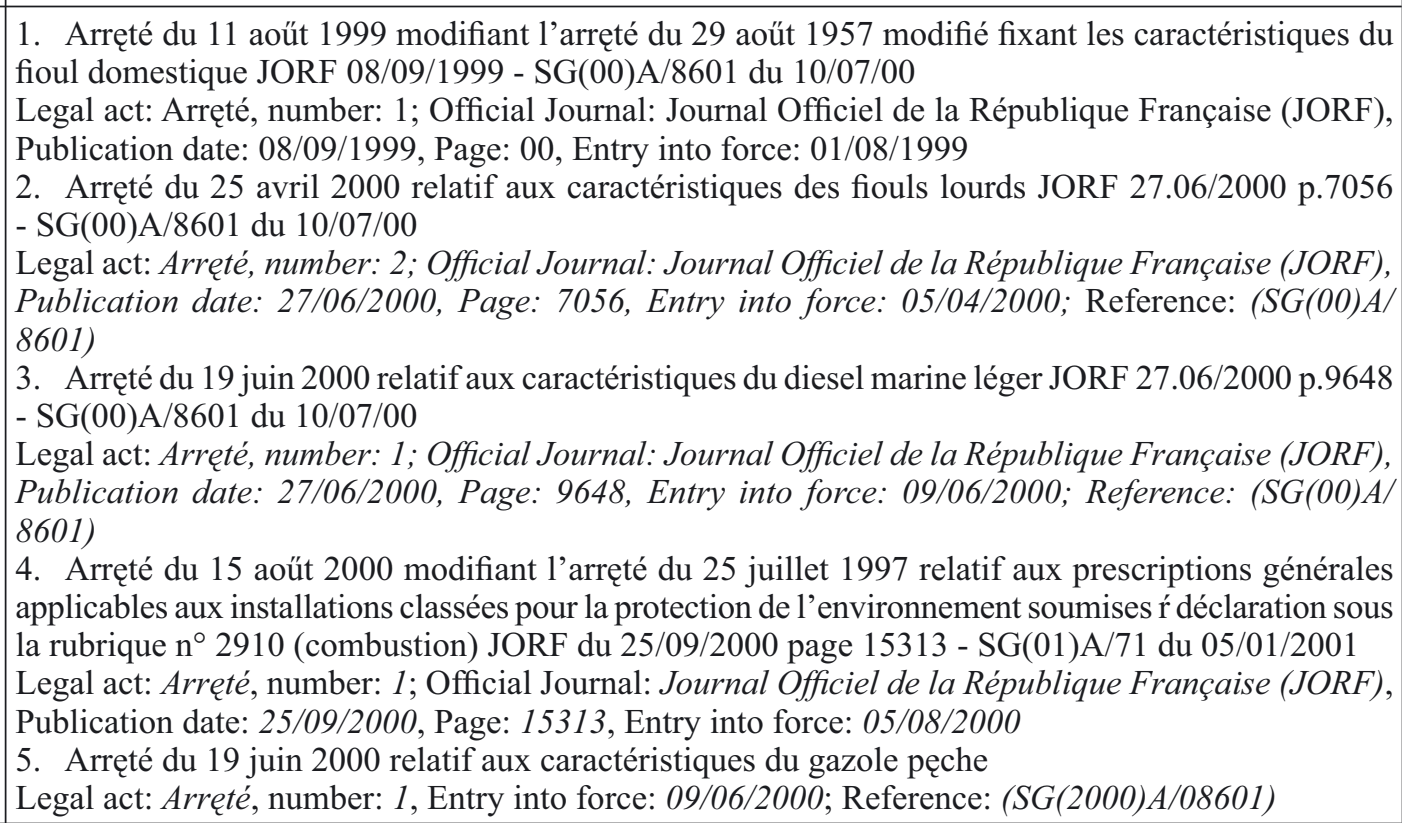 \\
\hline 7. & LATVIA & $\begin{array}{l}\text { 1. Par vides aizsardzību (Legal act: Likums; Official Journal: Latvijas Vēstnesis, number: } 33 \text {, } \\
\text { Publication date: 29/08/1991; Reference: (MNE(2003)50370) } \\
\text { 2. Administratīvo pārkāpumu kodekss (Legal act: Likums; Official Journal: Latvijas Vēstnesis, } \\
\text { Publication date: 01/07/1985; Reference: (MNE(2003)50416) } \\
\text { 3. Ministru Kabineta 2006.gada 26.septembra noteikumi Nr. } 801 \text { Noteikumi par sēra satura } \\
\text { ierobežošanu atseviškiem škidrās degvielas veidiem (Legal act: Ministru Kabineta noteikumi, number: } \\
\text { 801; Official Journal: Latvijas Vēstnesis, number: 161, Publication date: 10/10/2006, Entry into force: } \\
\text { 11/10/2006; Reference: (MNE(2006)56669) } \\
\text { 4. Likums "Par atbilstības novērtēšanu” (Legal act: Official Journal: Latvijas Vēstnesis, number: } \\
\text { 139 Likums;, Publication date: 20/08/1996; Reference: (MNE(2003)50596) } \\
\text { 5. Grozījumi likumā „Par piesārnojumu” (Legal act: Likums; Official Journal: Latvijas } \\
\text { Vēstnesis, number: 62, Publication date: 19/04/2006, Entry into force: 03/05/2006; Reference: } \\
\text { (MNE(2006)54960) }\end{array}$ \\
\hline
\end{tabular}




\begin{tabular}{|c|c|c|}
\hline Item & Country & National execution measures \\
\hline 7. & LATVIA & $\begin{array}{l}\text { 6. Ministru Kabineta 2004.gada 2.marta noteikumi Nr. } 125 \text { „Noteikumi par sēra satura ierobežošanu } \\
\text { noteiktiem šksidrās degvielas veidiem” (Legal act: Ministru Kabineta noteikumi; Official Journal: } \\
\text { Latvijas Vēstnesis, number: 37, Publication date: 09/03/2004; Reference: (MNE(2003)50860) } \\
\text { 7. Likums Par piesārnojumu (Legal act: Likums; Official Journal: Latvijas Vēstnesis, number: 51, } \\
\text { Publication date: 29/03/2001; Reference: (MNE(2003)50252) } \\
\text { 8. Ministru Kabineta 2002. gada 20. augusta noteikumi Nr. } 379 \text { „Kārtība, kādā novēršama, } \\
\text { ierobežojama un kontrolējama gaisu piesārnojošo vielu emisija no stacionāriem piesārnojuma } \\
\text { avotiem” (Legal act: Ministru Kabineta noteikumi; Official Journal: Latvijas Vēstnesis, number: } \\
\text { 122, Publication date 30/08/2002; Reference: (MNE(2003)50757). }\end{array}$ \\
\hline 8. & LITHUANIA & 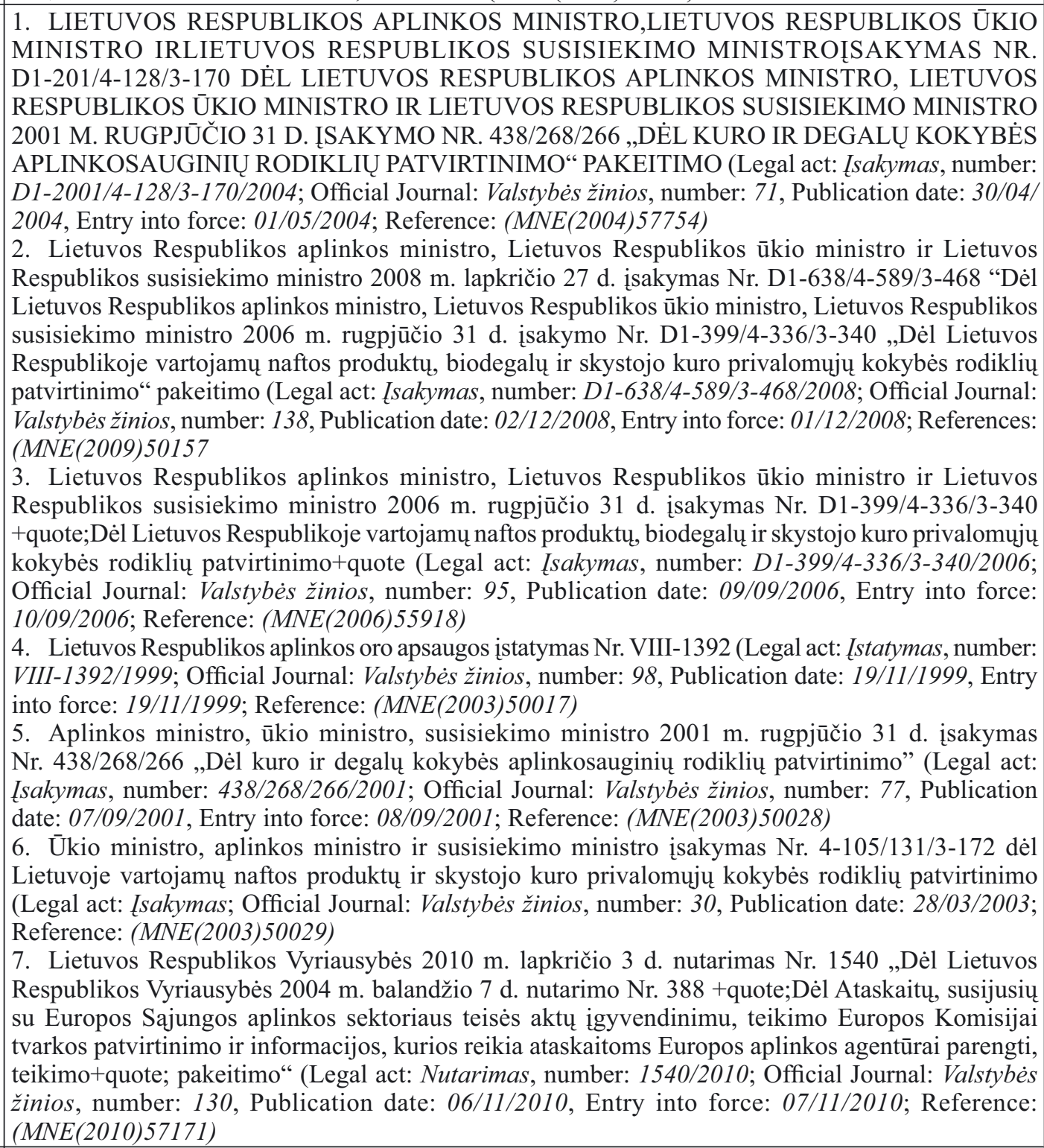 \\
\hline 9. & POLAND & 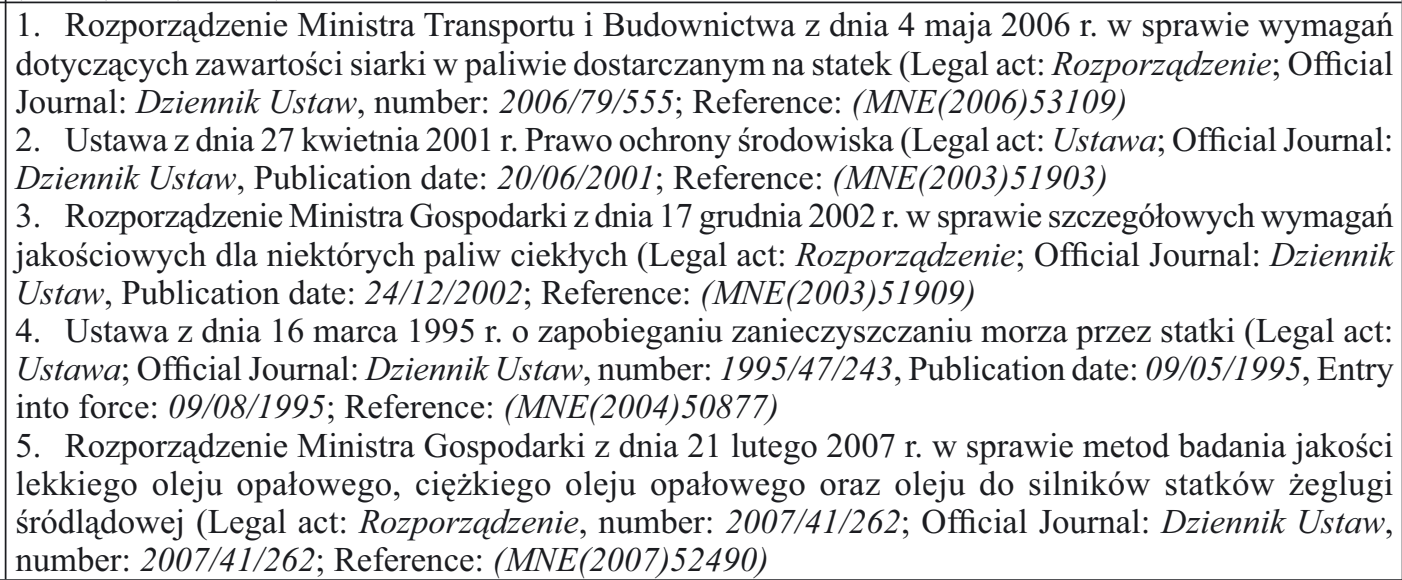 \\
\hline
\end{tabular}




\begin{tabular}{|c|c|l|}
\hline Item & Country & \multicolumn{1}{|c|}{ National execution measures } \\
\hline 9. & POLAND & $\begin{array}{l}\text { 6. Rozporzadzenie Ministra Gospodarki z dnia 21 lutego 2007 r. w sprawie sposobu pobierania } \\
\text { próbek lekkiego oleju opałowego, ciężkiego oleju opałowego oraz oleju do silników statków żeglugi } \\
\text { śródlądowej (Legal act: Rozporzadzenie, number: 2007/41/261; Official Journal: Dziennik Ustaw, } \\
\text { number: 2007/41/261; Reference: (MNE(2007)52489) } \\
\text { 7. Rozporządzenie Ministra Gospodarki z dnia 4 stycznia 2007 r. w sprawie wymagań jakościowych } \\
\text { dotyczacych zawartości siarki dla olejów oraz rodzajów instalacji i warunków, w których będa } \\
\text { stosowane ciężkie oleje opałowe (Legal act: Rozporzadzenie, number: 2007/4/30; Official Journal: } \\
\text { Dziennik Ustaw, number: 2007/4/30; Reference: (MNE(2007)50728) } \\
\text { 8. Ustawa z dnia 25 sierpnia 2006 r. o systemie monitorowania i kontrolowania jakości paliw (Legal } \\
\text { act: Ustawa, number: 2006/169/1200; Official Journal: Dziennik Ustaw, number: 2006/169/1200; } \\
\text { Reference: (MNE(2007)50623) }\end{array}$ \\
\hline $\mathbf{1 0 .}$ & SWEDEN & $\begin{array}{l}\text { 1. Miljöbalken 1998:908 (Legal act: Administrative measures) } \\
\text { 2. Förordningen 2000:372 om ändring i förordningen 1998:946 om svavelhaltigt bränsle (Legal act: } \\
\text { Administrative measures) }\end{array}$ \\
\hline
\end{tabular}

Following the entry into force of MARPOL Annex VI in May 2005 a new Directive 2005/33/EC (Directive 2005/33/EC of the European Parliament and of the Council of 6 July 2005 amending Directive 1999/32/EC, OJ L 191, 22.7.2005), was promulgated in July 2005, amending Directive No 32 adopted in 1999 (hereinafter referred to as the Sulphur Directive).

The Directive 2005/33/EC of the European Parliament and of the Council of $6^{\text {th }}$ July 2005, amending the Sulphur Directive, introduced, inter alia, the IMO concept of Sulphur Emission Control Areas (SECAs) and the associated stricter fuel standards. The maximum sulphur content of marine fuels was limited to a maximum of $1.5 \%$ for ships operating in the Baltic Sea as from 2006 and in the North Sea and the English Channel as from 2007. In addition, and in recognition of the need to further improve air quality for the protection of human health beyond the SECAs, some requirements that went beyond the IMO rules were introduced of which the most important are:

1. The obligation for ships at berth or anchorage in EU ports to use fuels containing maximum $0.1 \%$ sulphur;

2. The obligation for passenger ships on regular services to $\mathrm{EU}$ ports to use fuels containing a maximum sulphur content of $1.5 \%$;

3. The introduction of a possibility to test and use the emission abatement technologies.

Member States were obliged to transpose the Directive into national legislation by 11 August 2006.

The list of national enforcement measures in the exemplary EU Member States, covering the provisions of the Directive 2005/33/EC, are shown in the table below:

\begin{tabular}{|c|c|c|}
\hline Item & Country & National execution measures \\
\hline 1. & DENMARK & $\begin{array}{l}\text { 1. Bekendtgrrelse om svovlindholdet i faste og flydende bréndsler (Legal act: Bekendtgřrelse, number: } \\
\text { 1663; Official Journal: Lovtidende A, number: 1663; Reference: (MNE(2007)50430) }\end{array}$ \\
\hline 2. & GERMANY & $\begin{array}{l}\text { 1. 8. Schiffssicherheitsanpassungsverordnung (Legal act: Verordnung; Official Journal: } \\
\text { Bundesgesetzblatt Teil } 1 \text { (BGB 1), Publication date: 30/06/2006; Reference: (MNE(2006)54300) }\end{array}$ \\
\hline 3. & ESTONIA & 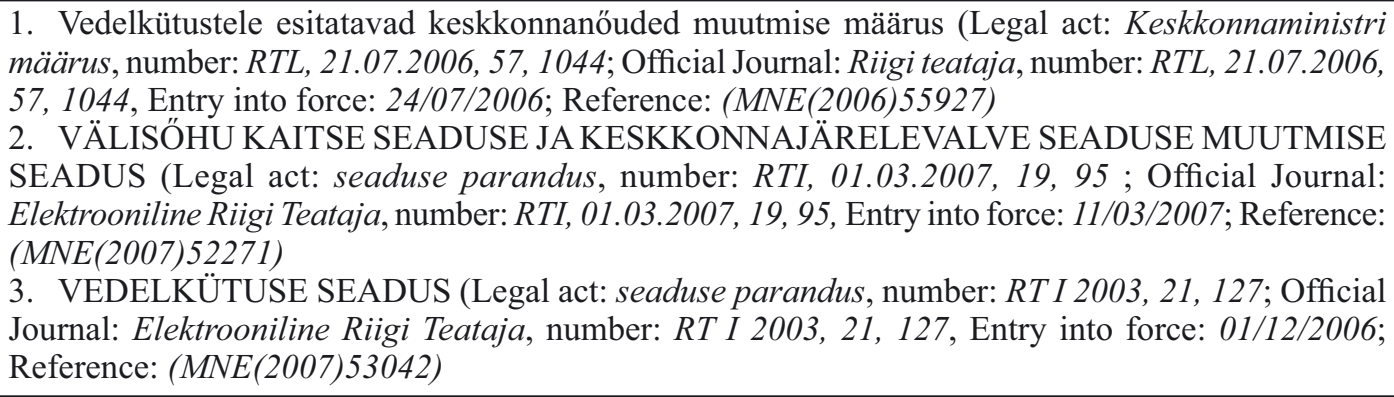 \\
\hline 4. & IRELAND & $\begin{array}{l}\text { 1. Sulphur Content of Heavy Fuel Oil, Gas Oil and Marine Fuels Regulations } 2008 \text {.SI } 119 \text { of } 2008 \text { Legal } \\
\text { act: Statutory Instrument (Regulation or Order), number: } 119 \text { of 2008; Official Journal: Iris Oifigiúl, } \\
\text { Publication date: } 02 / 05 / 2008 \text {, Entry into force: } 01 / 05 / 2008 \text {; Reference: (MNE(2008)52765) }\end{array}$ \\
\hline 5. & FINLAND & $\begin{array}{l}\text { 1. Concordance table (Legal act: Concordance table; Reference: (MNE(2006)55597) } \\
\text { 2. Valtioneuvoston asetus raskaan polttoöljyn, kevyen polttoöljyn ja meriliikenteessä käytettävän } \\
\text { kaasuöljyn rikkipitoisuudesta / Statsrldets förordning om svavelhalten i tung brännolja, lätt brännolja } \\
\text { och marin dieselbrännolja (689/2006) (Legal act: Valtioneuvoston asetus, number: 689/2006; Official } \\
\text { Journal: Suomen Saadoskokoelma (SK), number: } 689 \text {, Publication date: 08/08/2006, Page: } 02128 \text { - } \\
\text { 02131, Entry into force: } 11 / 08 / 2006 \text {; Reference: (MNE(2006)55594) } \\
\text { 3. Kaupparekisterilaki / Handelsregisterlag (129/1979) (Legal act: Laki, number: 129/1979; Official } \\
\text { Journal: Suomen Saadoskokoelma (SK), number: } 129 \text {; Reference: (MNE(2006)55595) } \\
\text { 4. Valtioneuvoston asetus aluksista aiheutuvan ympäristön pilaantumisen ehkäisemisestä/ Statsrídets } \\
\text { förordning om förhindrande av miljöförorening frln fartyg (635/1993) ja sen muutokset / och sina } \\
\text { förändringar: Valtioneuvoston asetukset / Statsrldets förordningar 1167/2003, 292/2005 ja 688/2006 } \\
\text { (Legal act: Valtioneuvoston asetus, number: 635/1993; Official Journal: Suomen Saadoskokoelma } \\
\text { (SK), number: 635; Reference: (MNE(2006)55592) }\end{array}$ \\
\hline
\end{tabular}




\begin{tabular}{|c|c|c|}
\hline Item & Country & National execution measures \\
\hline 5. & FINLAND & $\begin{array}{l}\text { 5. Laki aluksista aiheutuvan ympäristön pilaantumisen ehkäisemisestä / Lag om förhindrande av } \\
\text { miljöförorening frín fartyg (300/1979) ja sen muutokset / och sina förändringar: laki / lag 21.4.1995 } \\
\text { (696/1995), laki / lag 24.6.1999 (433/2000) ja/och laki / lag 18.3.2005 (238/2005) (Legal act: Laki, } \\
\text { number: 300/1979; Official Journal: Suomen Saadoskokoelma (SK), number: 300; Reference: } \\
\text { (MNE(2006)55587) }\end{array}$ \\
\hline 6. & SPAIN & $\begin{array}{l}\text { 1. Real Decreto } 1027 / 2006 \text {, de } 15 \text { de septiembre, por el que se modifica el Real Decreto } 61 / 2006 \text {, de } \\
31 \text { de enero, en lo relativo al contenido de azufre de los combustibles para uso marítimo } \\
\text { Legal act: Real Decreto, number: } 1027 / 2006 \text {; Official Journal: Boletín Oficial del Estado (B.O.E), } \\
\text { number: } 232 / 2006 \text {, Publication date: } 28 / 09 / 2006 \text {, Page: } 33889 \text {-33891, Entry into force: } 29 / 09 / 2006 \text {; } \\
\text { Reference: (MNE(2006)56332) }\end{array}$ \\
\hline 7. & LATVIA & $\begin{array}{l}\text { 1. Ministru Kabineta 2006.gada 26.septembra noteikumi Nr. } 801 \text { Noteikumi par sēra satura } \\
\text { ierobežošanu atseviškiem šķidrās degvielas veidiem (Legal act: Ministru Kabineta noteikumi, number: } \\
\text { 801; Official Journal: Latvijas Vēstnesis, number: 161, Publication date: 10/10/2006, Entry into force: } \\
\text { 11/10/2006; Reference: (MNE(2006)56669) } \\
\text { 2. Grozijumi likumā „Par piesārnojumu” (Legal act: Likums; Official Journal: Latvijas Vēstnesis, number: } \\
\text { 62, Publication date: 19/04/2006, Entry into force: 03/05/2006; Reference: (MNE(2006)54960) }\end{array}$ \\
\hline 8. & LITHUANIA & $\begin{array}{l}\text { 1. Lietuvos Respublikos aplinkos ministro, Lietuvos Respublikos ūkio ministro ir Lietuvos Respublikos } \\
\text { susisiekimo ministro } 2006 \mathrm{~m} \text {. rugpjūčio } 31 \text { d. isakymas Nr. D1-399/4-336/3-340 +quote;Dèl Lietuvos } \\
\text { Respublikoje vartojamų naftos produktu, biodegalų ir skystojo kuro privalomujų kokybès rodikliu } \\
\text { patvirtinimo+quote (Legal act: Isakymas, number: D1-399/4-336/3-340/2006; Official Journal: } \\
\text { Valstybès žinios, number: 95, Publication date: 09/09/2006, Entry into force: 10/09/2006; Reference: } \\
\text { (MNE(2006)55918) }\end{array}$ \\
\hline 9. & POLAND & 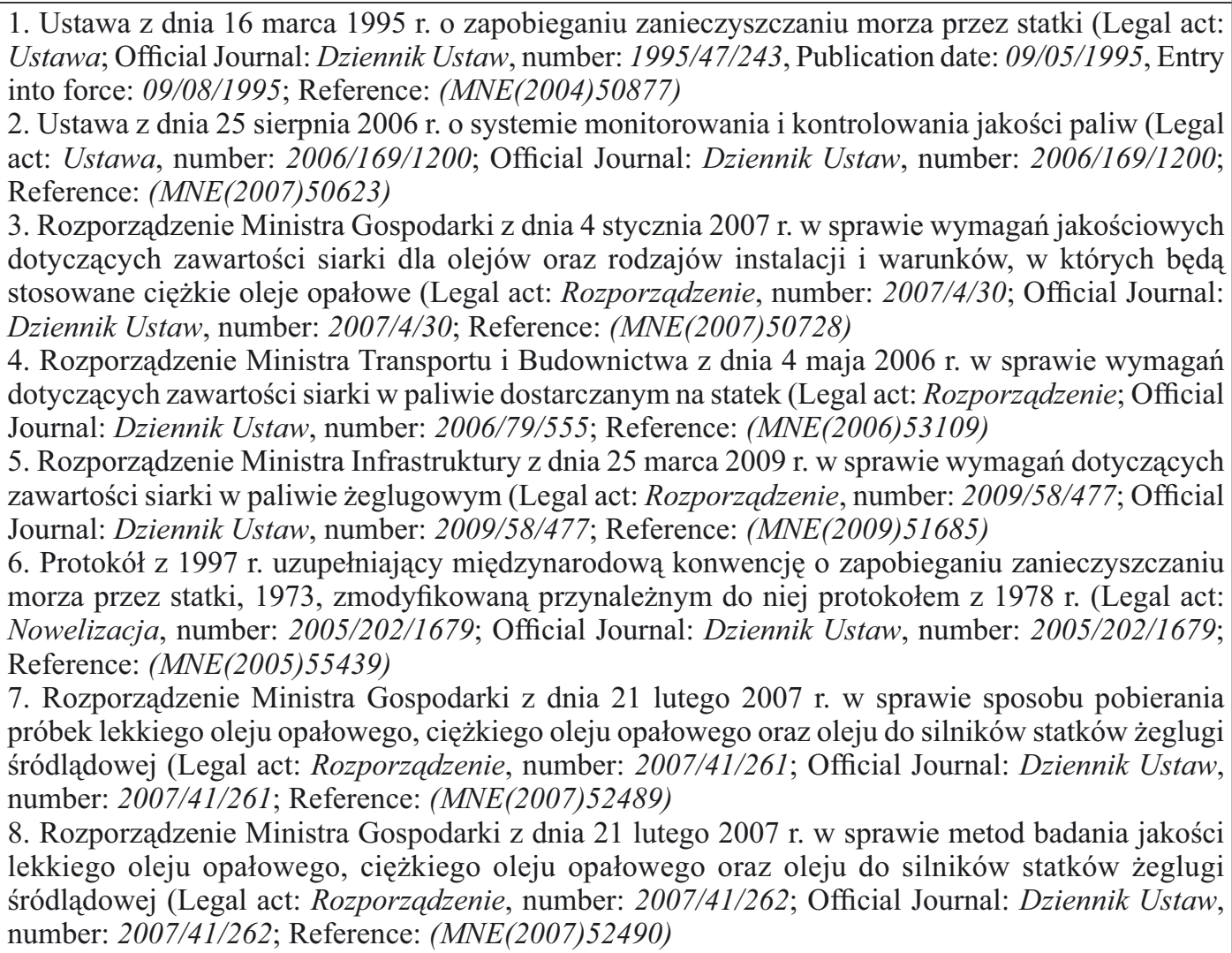 \\
\hline 10. & SWEDEN & $\begin{array}{l}\text { 1. Förordning (2006:1198) om ändring i förordningen (1998:946) om svavelhaltigt bränsle (Legal } \\
\text { act: Förordning, number: 2006:1198; Official Journal: Svensk författningssamling (SFS), number: } \\
\text { 2006:1198, Entry into force: 01/01/2007; Reference: (MNE(2006)58141) } \\
\text { 2. Förordning om ändring i förordningen (1998:946) omsvavelhaltigt bränsle (Legal act: Förordning; } \\
\text { Official Journal: Svensk författningssamling (SFS), Publication date: 09/11/2006, Entry into force: } \\
\text { 01/01/2007; Reference: (MNE(2006)57638) }\end{array}$ \\
\hline
\end{tabular}

The latest significant revision of the Sulphur Directive arises from the amendments to MARPOL Annex VI done in 2008, which imposed more stringent limits of sulphur content on fuels which are to be used in the Emission Control Areas. The EU rendered mandatory IMO rules on marine fuels through the Directive no 12, effective as of 17th December 2012, amending Sulphur Directive No 32 adopted in 1999 (Directive 2012/33/EU of the European Parliament and of the Council of 21 November 2012 amending Council Directive 1999/32/EC as regards the sulphur content of marine fuels). 
The key elements of the new directive are:

the sulphur limit in the Emission Control Areas (ECAs) is now $1.0 \%$ falling to $0.10 \%$ in 2015 (it was $1.5 \%$ under the EU sulphur directive prior to this amendment);

- the marine fuel used in the EU must meet the global $3.50 \%$ sulphur limit, except for on ships using an exhaust gas cleaning system operating in closed mode;

- a $0.50 \%$ sulphur limit will be implemented in all EU waters (outside Emission Control Areas (ECAs) by 2020, even if the IMO decides to delay the global limit;

- passenger ships operating outside ECAs but on regular service between EU ports continue to be subject to a $1.50 \%$ sulphur limit until 2020, when the EU-wide $0.50 \%$ sulphur limit applies;

ships at berth in EU ports are required to use only fuels with a maximum $0.1 \%$ sulphur content;

marine diesel oils sold in the EU must not exceed $1.50 \%$ sulphur (as before).

By $18^{\text {th }}$ June 2014 at the latest, Member States will have to amend their existing legislation on the quality of marine fuels to align it with the new Directive. The Directive provides legal certainty for the required investments by ship owners, port operators and refineries.

From 2015 onwards, Member States are asked to ensure that ships use fuels with a sulphur content of not more than $0.10 \%$ in the Baltic Sea and the North Sea including English Channel. Equivalent, compliance methods, such as exhaust cleaning systems, are accepted.

From 2020 onwards, ships operating in all other European Sea areas will have to use fuels with sulphur content of $0.50 \%$ or less.

The list of national enforcement measures in EU Member State, covering the provisions of the Directive 2012/33/EU, is shown in the table below:

\begin{tabular}{|c|c|l|}
\hline Item & Country & \multicolumn{1}{|c|}{ National execution measures } \\
\hline 1. SLOVAKIA & $\begin{array}{l}\text { 1. Vyhláška Ministerstva životného } \\
\text { prostredia Slovenskej republiky č. 231/ } \\
\text { 2013 Z. z. o informáciách podávaných } \\
\text { Európskej komisii, o požiadavkách } \\
\text { na vedenie prevádzkovej evidencie, } \\
\text { o údajoch oznamovaných do Národného } \\
\text { emisného informačného systému } \\
\text { a o súbore technicko-prevádzkových } \\
\text { parametrov a technicko-organizačných } \\
\text { opatrení } \\
\text { Legal act: vyhláška, number: 231/2013; } \\
\text { Official Journal: Zbierka zákonov SR, } \\
\text { number: 55, Publication date: } 22 / 08 \\
\text { 2013, Entry into force: 01/09/2013; } \\
\text { Reference: (MNE(2013)57747) }\end{array}$ \\
\hline
\end{tabular}

Council Directive 1999/32/EC of 26 April 1999 relating to a reduction in the sulphur content of certain liquid fuels lays down the maximum permitted sulphur content of heavy fuel oil, gas oil, marine gas oil and marine diesel oil used in the Union. Emissions from shipping due to the combustion of marine fuels with a high sulphur content contribute to air pollution in the form of sulphur dioxide and particulate matter, which harm human health and the environment, and contribute to acid deposition. Without the measures set out in this Directive, emissions from shipping would soon have been higher than emissions from all land-based sources. Air pollution caused by ships at berth is a major concern for many harbour cities when it comes to their efforts to meet the Union's air quality limit values. Under Directive 1999/32/EC, the Commission is to report to the European Parliament and the Council on the implementation of that Directive and may submit with its report proposals for amending it, in particular as regards the reduction of sulphur limits for marine fuel in SOx Emission Control Areas (SECAs), in accordance with the work of the International Maritime Organisation (IMO). In 2008, the IMO adopted a resolution to amend Annex VI of the Protocol of 1997 to amend the International Convention for the Prevention of Pollution from Ships, 1973, as modified by the Protocol of 1978 relating thereto (MARPOL), containing regulations for the prevention of air pollution from ships. The revised Annex VI to MARPOL entered into force on 1 July 2010. The revised Annex VI to MARPOL introduces, inter alia, stricter sulphur limits for marine fuel in SECAs (1.00\% as of 1 July 2010 and $0.10 \%$ as of 1 January 2015) as well as in sea areas outside SECAs (3.50\% as of 1 January 2012 and, in principle, $0.50 \%$ as of 1 January 2020). Most Member States are obliged, in accordance with their international commitments, to require ships to use fuel with a maximum sulphur content of $1.00 \%$ in SECAs as of 1 July 2010. In order to ensure coherence with international law as well as to secure proper enforcement of new globally established sulphur standards in the Union, Directive 1999/32/EC should be aligned with the revised Annex VI to MARPOL. In order to ensure a minimum quality of fuel used by ships either for fuel-based or technology-based compliance, the marine fuel the sulphur content of which exceeds the general standard of 3,50\% by mass should not be allowed for use in the Union, except for fuels supplied to ships using emission abatement methods operating in closed mode. Amendments to Annex VI to MARPOL regarding SECAs are possible under IMO procedures. In the event that further changes, including exemptions, are introduced with regard to the application of SECA limits in Annex VI to MARPOL, the Commission should consider any such changes and, where appropriate, without delay make the necessary proposal in accordance with the TFEU to fully align Directive 1999/32/EC with the IMO rules regarding SECAs. The introduction of any new emission control areas should be subject to the IMO process under Annex VI to MARPOL and should be underpinned by a well-founded case based on environmental and economic grounds and supported by scientific data. In accordance with regulation 18 of the revised Annex VI to MARPOL, Member States should endeavour to ensure the availability of marine fuels which comply with this Directive. In view of the global dimension of environmental politics and shipping emissions, ambitious emission standards should be set at a global level. Passenger ships operate mostly in ports or close to coastal areas and their impacts on human health and the environment are significant. In order to improve the air quality around ports and coasts, those ships are required to use marine fuel with a maximum sulphur content of $1.50 \%$ until stricter sulphur standards apply to all ships in territorial seas, exclusive economic zones and pollution control zones of Member States.

Complying with the low sulphur limits for marine fuels, particularly in SECAs, can result in a significant increase in the price of such fuels, at least in the short term, and can have a negative effect on the competitiveness of short sea shipping in comparison with other transport modes, as well as on the competitiveness of the industries in the countries bordering SECAs. Suitable solutions are necessary in order to reduce compliance costs for the affected industries, such as allowing for alternative, more cost-effective methods of compliance than fuel-based compliance and providing support, where necessary. Based inter alia on reports from Member States, the Commission will closely monitor the impacts of the shipping 
sector's compliance with the new fuel quality standards, particularly with respect to possible modal shift from sea to land-based transport and will, if appropriate, propose proper measures to counteract such a trend.

The costs of the new requirements to reduce sulphur dioxide emissions could result in modal shift from sea to land-based transport and could have negative effects on the competitiveness of the industries. The Commission should make full use of instruments such as Marco Polo and the transEuropean transport network to provide targeted assistance so as to minimise the risk of modal shift. Member States may consider it necessary to provide support to operators affected by this Directive in accordance with the applicable State aid rules.

\section{CONTACT WITH THE AUTHOR}

Beata Madejska, M. Sc.

lawyer specialized in the EU legal regulations mobile: +48606273740

e-mail: beata.madejska@interia.pl 